tive jaundice as a complication of benign ulcer of the pylorus or duodenum (especially the second part) was discussed.

MR. G. W. SINCLAIR reported 'An unusual example of the blind loop syndrome', resulting from chronic incomplete obstruction of the ileum caused by a band running from the tip of the appendix to the parietes. The simple surgical relief of this condition resulted in a rapid and dramatic return to good health and normal nutrition.

\section{Symposium in Marseilles}

A number of European clinicians and pathologists met from 25 to 27 April 1963 in Marseilles under the Chairmanship of Dr. Henri Sarles to discuss the aetiology and pathological anatomy of chronic pancreatitis. ${ }^{1}$ Since it was early apparent that the term 'chronic pancreatitis' was being variously interpreted, a working party met to draft a classification which was later accepted unanimously by the attending members. It is hoped that the general adoption of this classification at least for the present will enable comparison and appraisal of communications made by workers from different centres.

Pancreatitis can be classified clinically: 1 Acute pancreatitis; 2 relapsing acute pancreatitis. In the two acute forms clinical and biological restitution of the pancreas takes place if the primary cause or factors are eliminated. It is unusual for acute pancreatitis to develop into chronic pancreatitis, but this may occur. 3 Relapsing chronic pancreatitis, defined as chronic pancreatitis with acute exacerbations; 4 chronic pancreatitis. In the two chronic forms residual pancreatic damage, either anatomical or functional, persists even if the primary cause or factors are eliminated. Chronic pancreatitis may result from relapsing chronic pancreatitis or may manifest itself from the first as a chronic disease. Occasionally it may follow the acute form. The distinction between the two chronic groups is clinical and not morphological.

The Marseilles Symposium was primarily concerned with the aetiology and pathological anatomy of the chronic groups and it was not intended to explore the correlation between anatomical and functional changes.

\section{MORPHOLOGY}

The morbid anatomical picture of chronic pancreatitis is characterized by irregular sclerosis with destruction and loss of exocrine parenchyma, either focal, segmental, or diffuse. These features may be associated with variable degrees of dilatation of segments of the duct system. Thus dilatation of the duct of Wirsung and of the smaller ductules may occur together or independently. No

${ }^{1}$ The proceedings of the Symposium will be published in full by Karger in Bibliotheca Gastroenterologia. obvious cause of the dilatation may be found but most often it is associated with strictures or stones. All types of inflammatory cells may be present in varying degree as well as oedema, necrosis, and abscess formation. Cysts and pseudo-cysts which may or may not communicate with the ducts are not uncommon. In comparison with the destruction of the acini, the islets of Langerhans are relatively well preserved. The incidence of calcification in chronic pancreatitis is variable; if present it is almost invariably intraductal (stones) and but rarely parenchymal (calcification). All these histological features may be seen in cases of chronic pancreatitis of differing aetiology.

\section{AETIOLOGY}

The incidence of chronic pancreatitis in the different European countries represented at this Symposium varies considerably and is apparently directly related to the incidence of alcoholic pancreatitis. This latter leads frequently to a calcified form of pancreatitis. The coincidence of cirrhosis of the liver with alcoholic chronic pancreatitis was rare in the French series. Cholelithiasis is commonly associated with the acute forms of pancreatitis but less frequently accompanies the chronic forms. Other lesions which may obstruct the main pancreatic ducts and lead to chronic pancreatitis are Vaterian stenosis (Oddite), traumatic stricture, stone formation, cancer, and duodenal obstruction. Among rarer causes of chronic pancreatitis are hereditary, metabolic, nutritional, endocrine, and vascular factors as well as drug addiction. In all but the alcoholic, hereditary, and endocrine groups calcification is rare.

H.T.H.

\section{Indian Society of Gastroenterology}

The annual meeting was held in Calcutta in January 1963. Dr. Sangham Lal (New Delhi) was in the Chair, and Dr. W. I. Card was the guest of honour. The scientific sessions contained papers on a wide range of topics, and in addition there were two symposia, one on cirrhosis of the liver and the other on pancreatitis. In the presidential address Dr. Sangham Lal took as his subject the problem of peptic ulcer in India and its medical and surgical management. At the general meeting Dr. P. N. Chuttani (Chandigarh) was elected President.

The Asian Congress of Gastroenterology will be held at Chandigarh from 27 to 29 January 1964. Observers from the British Society of Gastroenterology will be welcome. Enquiries should be addressed to B. L. Talwar, M.S. (Bom.), F.R.C.S. (Eng.), Organizing Secretary, Postgraduate Institute of Medicine and Research, Chandigarh, Punjab, India. 